\title{
TOPOLOGICAL NOETHERIANITY FOR ALGEBRAIC REPRESENTATIONS OF INFINITE RANK CLASSICAL GROUPS
}

\section{R. H. EGGERMONT*}

Department of Mathematics and Computer Science

Eindhoven University of Technology Eindhoven, Netherlands r.h.eggermont@tue.nl

\section{A. SNOWDEN**}

Department of Mathematics

University of Michigan

Ann Arbor, USA

asnowden@umich.edu

\begin{abstract}
Draisma recently proved that polynomial representations of $\mathbf{G L}_{\infty}$ are topologically noetherian. We generalize this result to algebraic representations of infinite rank classical groups.
\end{abstract}

\section{Introduction}

\subsection{Background}

In recent years, a number of novel noetherianity results have been discovered and exploited; for but a few examples, see [AH], [Co], [Co2], [CEF], [DE], [DES], [DK], $[\mathrm{Eg}],[\mathrm{HS}],[\mathrm{NSS}],[\mathrm{PSa}],[\mathrm{Sn}],[\mathrm{SS} 2]$. It is not yet clear where the ultimate line between noetherianity and non-noetherianity lies: of the reasonable structures to consider, some are known to be noetherian and some not, and in between is a vast unknown territory.

Recently, Draisma [Dr] proved a breakthrough result claiming a large tract of the unknown for the noetherian side. To state it, we must recall some terminology. Fix an algebraically closed field $\mathbf{k}$ and let $\mathbf{G L}=\bigcup_{n \geq 1} \mathbf{G L}_{n}(\mathbf{k})$. (One can identify $\mathbf{G L}_{n}$ with a subgroup of $\operatorname{Aut}\left(\mathbf{k}^{\infty}\right)$ and take the union within this ambient group. Alternatively, one can regard this union as the direct limit with respect to the natural inclusions $\mathbf{G L}_{n} \rightarrow \mathbf{G} \mathbf{L}_{n+1}$.) A representation of $\mathbf{G L}$ is called polynomial if it is a subquotient of a finite direct sum of tensor products of the standard representation $\mathbf{V}=\bigcup_{n \geq 1} \mathbf{k}^{n}$. (Note that in other settings, one allows infinite sums of tensor powers, but in this paper we restrict to finite sums.) If $\mathbf{E}$ is a polynomial representation then the dual space $\widehat{\mathbf{E}}$ is canonically identified with the $\mathbf{k}$-points of the spectrum of the ring $\operatorname{Sym}(\mathbf{E})$, and in this way inherits a Zariski topology.

DOI: $10.1007 / \mathrm{s} 00031-021-09656-\mathrm{x}$

${ }^{*} \mathrm{RE}$ was supported by the Dutch Research Council (NWO) via a Veni grant on the topic of 'Stability and structure in infinite-dimensional spaces'.

${ }^{* *}$ AS was supported by NSF grants DMS-1303082 and DMS-1453893 and a Sloan Fellowship.

Received March 23, 2020. Accepted January 7, 2021.

Published online May 19, 2021.

Corresponding Author: R. H. Eggermont, e-mail: r.h.eggermont@tue.nl 
Recall that if a group $G$ acts on a space $X$ (such as GL on $\widehat{\mathbf{E}}$ ) then we say that $X$ is topologically $G$-noetherian if every descending chain of $G$-stable closed subsets of $X$ stabilizes. We can now state Draisma's theorem:

Theorem 1 (Draisma). Let $\mathbf{E}$ be a polynomial representation of GL. Then $\widehat{\mathbf{E}}$ is topologically GL-noetherian.

This result has already found application: Erman-Sam-Snowden [ESS] have combined it with the resolution of Stillman's conjecture by Ananyan-Hochster [AH2] to establish a vast generalization of Stillman's conjecture.

\subsection{The main theorem}

Draisma's theorem elicits a few natural follow-up questions. Does noetherianity hold for any non-polynomial representations of GL? And what about representations of similar groups, such as the infinite orthogonal group? The purpose of this paper is to provide some answers to these questions.

Let $\mathbf{O}=\bigcup_{n \geq 1} \mathbf{O}_{n}(\mathbf{k})$ and $\mathbf{S p}=\bigcup_{n \geq 1} \mathbf{S p}_{2 n}(\mathbf{k})$ be respectively the infinite orthogonal group and the infinite symplectic group, where the unions are taken in the same sense as for GL.

Note that $\mathbf{O}_{n}(\mathbf{k})$, respectively $\mathbf{S p}_{2 n}(\mathbf{k})$, is defined as the elements of $\mathbf{G L}_{n}(\mathbf{k})$, respectively $\mathbf{G L}_{2 n}(\mathbf{k})$, that fix a given symmetric bilinear form on $\mathbf{k}^{n}$, respectively a given symplectic bilinear form on $\mathbf{k}^{2 n}$. The actual forms used are of low importance since they give the same group up to isomorphism. We do require that the inclusions behave well with respect to these forms; the form defined on a larger space should extend the form defined on the included space. In particular, this induces a bilinear form on the relevant direct limit.

We say that a representation of $\mathbf{O}$ or $\mathbf{S p}$ is algebraic if it appears as a subquotient of a finite direct sum of tensor powers of the standard representation $\mathbf{V}$. Let $\mathbf{V}_{*}=\bigcup_{n>1}\left(\mathbf{k}^{n}\right)^{*}$, the so-called restricted dual of $\mathbf{V}$. The group $\mathbf{G L}$ acts on $\mathbf{V}_{*}$. A representation of $\mathbf{G L}$ is called algebraic if it appears as a subquotient of a finite direct sum of representations of the form $\mathbf{V}^{\otimes n} \otimes \mathbf{V}_{*}^{\otimes m}$. We note that $\mathbf{V} \cong$ $\mathbf{V}_{*}$ as representations of $\mathbf{O}$ and $\mathbf{S p}$, so one does not get a larger category of representations by using $\mathbf{V}_{*}$. In characteristic 0 , algebraic representations of $\mathbf{G L}$, $\mathbf{O}$, and $\mathbf{S p}$ have been studied in [DPS], [PSe], [PSt], [SS].

The main theorem of this paper is:

Theorem 2. Let $\mathbf{G} \in\{\mathbf{O}, \mathbf{S p}, \mathbf{G L}\}$ and let $\mathbf{E}$ be an algebraic representation of $\mathbf{G}$. Then $\widehat{\mathbf{E}}$ is topologically $\mathbf{G}$-noetherian.

In $\S 5$, we show that certain very similar looking statements are false. It would be interesting to see if this theorem has any applications in commutative algebra along the lines of [ESS]. We note, however, that [ESS] makes use of the kind of statements found in $\S 5$ that are true in the case of polynomial representations, but false for algebraic representations.

Acknowledgements. We thank Steven Sam for helpful discussions. We thank the anonymous reviewers for their helpful suggestions and corrections. 


\subsection{Possible generalizations}

There are two directions in which Theorem 2 might be generalized. First, one might hope for a statement at the level of ideals. That is, suppose that $\mathbf{E}$ is an algebraic representation of $\mathbf{G}$ (for $\mathbf{G}$ as in the theorem), and let $A=\operatorname{Sym}(\mathbf{E})$. The theorem is equivalent to the statement that any ascending chain of $\mathbf{G}$-stable radical ideals in $A$ stabilizes. A plausible stronger statement is that every ascending chain of $\mathbf{G}$ stable ideals stabilizes. More generally, if $\mathbf{E}^{\prime}$ is a second algebraic representation, one might hope that every ascending chain of G-stable submodules of $A \otimes \mathbf{E}^{\prime}$ stabilizes.

Second, one might hope to extend topological noetherianity to certain analogs of algebraic representations. Let $A=\operatorname{Sym}\left(\operatorname{Sym}^{2}\left(\mathbf{C}^{\infty}\right)\right)$, equipped with its natural action of GL. One can then consider the category $\operatorname{Mod}_{A}$ of $A$-modules equipped with a compatible polynomial action of GL. Let $\operatorname{Mod}_{A}^{\text {tors }}$ be the Serre subcategory of torsion modules. It is known [NSS] that the quotient category $\operatorname{Mod}_{A} / \operatorname{Mod}_{A}^{\text {tors }}$ is equivalent to the category of algebraic representations of $\mathbf{O}$ (as a tensor category). Thus Theorem 2 in the case $\mathbf{G}=\mathbf{O}$ can be stated as: every finitely generated algebra in the category $\operatorname{Mod}_{A} / \operatorname{Mod}_{A}^{\text {tors }}$ is topologically noetherian. It seems plausible that this statement might hold true for any $A$ of the form $\operatorname{Sym}(\mathbf{E})$, with $\mathbf{E}$ a polynomial representation of GL.

\subsection{Outline}

In $\S 2$, we show that it suffices to prove Theorem 2 for any one of the three groups. In $\S 3$, we go over some preliminary material. The main theorem (in the general linear case) is proved in $\S 4$. In $\S 5$, we discuss counterexamples to certain variants of the main theorem.

\section{Reduction to the general linear case}

We now show that it suffices to prove Theorem 2 in any one of the three cases. Later, we will prove the theorem in the general linear case.

Lemma 3. Suppose that $\mathbf{G}, \mathbf{H} \in\{\mathbf{G L}, \mathbf{S p}, \mathbf{O}\}$ and that $\mathbf{H} \rightarrow \mathbf{G}$ is a homomorphism such that the standard representation of $\mathbf{G}$ pulls back to an algebraic representation of $\mathbf{H}$. Suppose also that Theorem 2 holds for $\mathbf{H}$. Then Theorem 2 holds for $\mathbf{G}$.

Proof. The hypothesis implies that any algebraic representation of $\mathbf{G}$ pulls back to an algebraic representation of $\mathbf{H}$. Let $\mathbf{E}$ be an algebraic representation of $\mathbf{G}$. Suppose that $Z_{\bullet} \subset \widehat{\mathbf{E}}$ is a descending chain of $\mathbf{G}$-stable closed subsets. Then it is also a descending chain of $\mathbf{H}$-stable closed subsets, and thus stabilizes. Thus $\widehat{\mathbf{E}}$ is topologically G-noetherian.

Proposition 4. If Theorem 2 holds for any one of the three groups then it holds for the other two.

Proof. Suppose the theorem holds for GL and $\operatorname{char}(\mathbf{k}) \neq 2$. Consider the representation $\mathbf{V} \oplus \mathbf{V}_{*}$ of $\mathbf{G L}$. This representation carries both a symmetric form and 
a symplectic form, defined by the formulas $\left(v+\lambda, v^{\prime}+\lambda^{\prime}\right)=\lambda\left(v^{\prime}\right) \pm \lambda^{\prime}(v)$. These forms are GL-invariant and thus give group homomorphisms $\mathbf{G L} \rightarrow \mathbf{O}$ and $\mathbf{G L} \rightarrow$ Sp. (In a little more detail: let $\mathbf{V}_{n}=\mathbf{k}^{n}$. Then the space $\mathbf{V}_{n} \oplus \mathbf{V}_{n}^{*}$ carries similar forms, and we have a natural embedding $\mathbf{G L}_{n} \rightarrow \mathbf{G L}\left(\mathbf{V}_{n} \oplus \mathbf{V}_{n}^{*}\right)$ whose image is contained in the elements of $\mathbf{G L}\left(\mathbf{V}_{n} \oplus \mathbf{V}_{n}^{*}\right)$ that are invariant under these forms. We thus obtain homomorphisms $\mathbf{G L} \mathbf{L}_{n} \rightarrow \mathbf{O}_{2 n}$ and $\mathbf{G} \mathbf{L}_{n} \rightarrow \mathbf{S p}_{2 n}$. The direct limits of these maps are the maps $\mathbf{G L} \rightarrow \mathbf{O}$ and $\mathbf{G L} \rightarrow$ Sp.) These homomorphisms have the property that the standard representation of $\mathbf{O}$ or $\mathbf{S p}$ pulls back to the representation $\mathbf{V} \oplus \mathbf{V}_{*}$ of $\mathbf{G L}$. Thus Theorem 2 holds for $\mathbf{O}$ and $\mathbf{S p}$ by Lemma 3 .

If $\operatorname{char}(\mathbf{k})=2$ then a similar argument works. The bilinear form defined in the previous paragraph is alternating in characteristic 2 (i.e., it satisfies $(v+\lambda, v+\lambda)=$ $0)$ and thus yields a map $\mathbf{G L} \rightarrow \mathbf{S p}$ that again allows us to apply Lemma 3 . The representation $\mathbf{V} \oplus \mathbf{V}_{*}$ also admits a quadratic form defined by $(v, \lambda) \mapsto \lambda(v)$, which affords a homomorphim $\mathbf{G L} \rightarrow \mathbf{O}$ to which we can apply Lemma 3.

Now suppose that Theorem 2 holds for $\mathbf{O}$. Applying Lemma 3 to the inclusion $\mathbf{O} \rightarrow \mathbf{G L}$, we see that Theorem 2 holds for GL. Appealing to the previous two paragraphs, we thus see that Theorem 2 holds for $\mathbf{S p}$ as well. Similarly, if Theorem 2 holds for $\mathbf{S p}$ then we get it for $\mathbf{G L}$ and then $\mathbf{O}$.

A similar argument is used in the following proposition, which we also require. A representation of $\mathbf{G L} \times \mathbf{G L}$ is polynomial if it appears as a subquotient of a finite sum of representations of the form $\mathbf{V}^{\otimes n} \otimes \mathbf{V}^{\otimes m}$.

Proposition 5. Let $\mathbf{E}$ be a polynomial representation of $\mathbf{G L} \times \mathbf{G L}$. Then $\widehat{\mathbf{E}}$ is topologically $\mathbf{G L} \times \mathbf{G L}$ noetherian.

Proof. Consider the diagonal copy of $\mathbf{G L}$ in $\mathbf{G L} \times \mathbf{G L}$. The restriction $\left.\mathbf{E}\right|_{\mathbf{G L}}$ is then polynomial: indeed, if $\mathbf{E}$ is a subquotient of $\bigoplus_{i=1}^{k} \mathbf{V}^{\otimes n_{i}} \otimes \mathbf{V}^{\otimes m_{i}}$ then $\left.\mathbf{E}\right|_{\mathbf{G L}}$ is a subquotient of $\bigoplus_{i=1}^{k} \mathbf{V}^{\otimes\left(n_{i}+m_{i}\right)}$. Since $\left.\widehat{\mathbf{E}}\right|_{\mathbf{G L}}$ is topologically noetherian by Draisma's theorem, the result follows.

\section{Preliminaries}

\subsection{Spaces of matrices}

Let $\widehat{\mathbf{M}}$ be the set of matrices $\left(a_{i, j}\right)_{i, j \in \mathbf{N}}$ with $a_{i, j} \in \mathbf{k}$. Let $\mathbf{M} \subset \widehat{\mathbf{M}}$ be the subset consisting of matrices with only finitely many non-zero entries. We have a trace pairing

$$
\langle,\rangle: \widehat{\mathbf{M}} \times \mathbf{M} \rightarrow \mathbf{k}, \quad\langle A, B\rangle=\operatorname{tr}\left(A^{t} B\right)
$$

that identifies $\widehat{\mathbf{M}}$ with the linear dual of the space $\mathbf{M}$. We let $\mathbf{M}_{n} \subset \mathbf{M}$ be the set of matrices $\left(a_{i, j}\right)$ with $a_{i, j}=0$ for $i>n$ or $j>n$. We define $\widehat{\mathbf{M}}_{n}$ similarly, but regard it as a quotient of $\widehat{\mathbf{M}}$. Thus $\mathbf{M}$ is the union of the $\mathbf{M}_{n}$ and $\widehat{\mathbf{M}}$ is the inverse limit of the $\widehat{\mathbf{M}}_{n}$.

Let $\mathbf{U}, \mathbf{L} \subset \mathbf{M}$ and $\widehat{\mathbf{U}}, \widehat{\mathbf{L}} \subset \widehat{\mathbf{M}}$ be the spaces of upper-triangular and lowertriangular matrices. The trace pairing identifies $\widehat{\mathbf{L}}$ with the linear dual of $\mathbf{L}$ and $\widehat{\mathbf{U}}$ 
with the dual of $\mathbf{U}$. One cannot multiply arbitrary elements of $\widehat{\mathbf{M}}$, as this would typically involve infinite sums. However, the product $A B$ is defined for $A \in \widehat{\mathbf{L}}$ and $B \in \widehat{\mathbf{M}}$, or for $A \in \widehat{\mathbf{M}}$ and $B \in \widehat{\mathbf{U}}$. We define $\mathbf{L}_{n}, \mathbf{U}_{n}, \widehat{\mathbf{L}}_{n}$, and $\widehat{\mathbf{U}}_{n}$ in the obvious ways.

We let $\mathbf{V}_{n}=\mathbf{k}^{n}$ and $\mathbf{V}=\bigcup_{n \geq 1} \mathbf{V}_{n}$. We let $\widehat{\mathbf{V}}$ and $\widehat{\mathbf{V}}_{n}$ be the dual spaces to $\mathbf{V}$ and $\mathbf{V}_{n}$, so that $\widehat{\mathbf{V}}$ is the inverse limit of the spaces $\widehat{\mathbf{V}}_{n}$.

\subsection{Polynomial representations}

Let $\mathbf{E}$ be a polynomial representation of $\mathbf{G L}$. Then the action of $\mathbf{G L}_{n} \subset \mathbf{G L}$ extends uniquely to an action of the monoid $\mathbf{M}_{n}$, and these assemble to an action of $\mathbf{M}$. Furthermore, the action of $\mathbf{U}$ on the dual $\widehat{\mathbf{E}}$ extends uniquely to a continuous action of $\widehat{\mathbf{U}}$, where continuous means that for fixed $v \in \widehat{\mathbf{E}}$ the quantity $u v$ depends only on the projection of $u$ to $\widehat{\mathbf{U}}_{n}$, for some $n$ depending only on $v$. This is easy to see when $\mathbf{E}=\mathbf{V}$ : the point is that, if $e_{i}$ denotes the $i$ th basis vector of $\mathbf{V}$, then $u e_{i}^{*}$ only depends on the top left $i \times i$ block of $u$. The action of $\mathbf{L}$ on $\widehat{\mathbf{E}}$ does not similarly extend to an action of $\widehat{\mathbf{L}}$.

We can then equivalently think of the representation $\mathbf{E}$ as a polynomial functor $\underline{\mathbf{E}}$ on the category of $\mathbf{k}$-vector spaces. We let $\mathbf{E}_{n}$ be the value of the functor $\underline{\mathbf{E}}$ on $\mathbf{k}^{n}$, so that $\mathbf{E}$ itself is identified with the union of the $\mathbf{E}_{n}$. We let $\widehat{\mathbf{E}}$ and $\widehat{\mathbf{E}}_{n}$ be the linear duals of $\mathbf{E}$ and $\mathbf{E}_{n}$, so that $\widehat{\mathbf{E}}$ is the inverse limit of the $\widehat{\mathbf{E}}_{n}$. We note that $\mathbf{E}_{n} \subset \mathbf{E}$ is stable under the action of $\mathbf{U}$ and $\widehat{\mathbf{U}}$. It follows that the projection map $\widehat{\mathbf{E}} \rightarrow \widehat{\mathbf{E}}_{n}$ is compatible with the action of $\widehat{\mathbf{U}}$.

For the purposes of this paper, we really only need to consider E's that are finite sums of tensor powers of $\mathbf{V}$. In this case, one does not need to think about polynomial functors: the space $\mathbf{E}_{n}$ is then the corresponding sum of tensor powers of $\mathbf{V}_{n}$.

We identify $\widehat{\mathbf{E}}$ with the spectrum of the $\operatorname{ring} \operatorname{Sym}(\mathbf{E})$ (or more accurately, the k-points of the spectrum), and equip it with the Zariski topology. By definition, a regular function $f$ on $\widehat{\mathbf{E}}$ is an element of $\operatorname{Sym}(\mathbf{E})$. Since $\mathbf{E}$ is the union of the $\mathbf{E}_{n}$, it follows that $f$ belongs to $\operatorname{Sym}\left(\mathbf{E}_{n}\right)$ for some $n$; we say that $f$ has level $n$. This implies that $f$ factors through the projection $\widehat{\mathbf{E}} \rightarrow \widehat{\mathbf{E}}_{n}$.

\section{The general linear case}

Let $\mathbf{G}=\mathbf{G L} \times \mathbf{G L}$, and let $\mathbf{H}$ be the subgroup of elements of the form $\left(g,{ }^{t} g^{-1}\right)$. Of course, $\mathbf{H}$ is isomorphic to $\mathbf{G L}$. By definition, every algebraic representation of $\mathbf{H}$ is a subquotient of $\left.\mathbf{E}\right|_{\mathbf{H}}$ for some polynomial representation $\mathbf{E}$ of $\mathbf{G}$, so it suffices to prove noetherianity of $\left.\widehat{\mathbf{E}}\right|_{\mathbf{H}}$ for all such $\mathbf{E}$. We therefore fix $\mathbf{E}$ for the rest of this section, and prove that $\left.\widehat{\mathbf{E}}\right|_{\mathbf{H}}$ is noetherian.

Let $\mathbf{G}$ act on $\mathbf{M}$ by the formula $(g, h) \cdot A=g A^{t} h$. The dual action of $\mathbf{G}$ on $\widehat{\mathbf{M}}$ is given by the formula $(g, h) \cdot A={ }^{t} g^{-1} A h^{-1}$. Let $I \in \widehat{\mathbf{M}}$ be the identity matrix. Note that the stabilizer of $I$ in $\mathbf{G}$ is exactly $\mathbf{H}$. Given an $\mathbf{H}$-stable closed subset 
$Z$ of $\widehat{\mathbf{E}}$, let $Z^{+}$be the closure of the $\mathbf{G}$-orbit of the set $\{I\} \times Z$ in $\widehat{\mathbf{M}} \times \widehat{\mathbf{E}}$. (The topology on the product is the Zariski topology on the product of schemes, which is not the product topology.) We will prove:

Proposition 6. Let $Z$ be an $\mathbf{H}$-stable closed subset of $\widehat{\mathbf{E}}$. Then we have the equality $\{I\} \times Z=(\{I\} \times \widehat{\mathbf{E}}) \cap Z^{+}$.

Before proving the proposition, we note an important consequence.

Corollary 7. The function $Z \mapsto Z^{+}$defines an order-preserving injection

$$
\{\mathbf{H} \text {-stable closed subsets of } \widehat{\mathbf{E}}\} \rightarrow\{\mathbf{G} \text {-stable closed subsets of } \widehat{\mathbf{M}} \times \widehat{\mathbf{E}}\} \text {. }
$$

In particular, $\widehat{\mathbf{E}}$ is topologically $\mathbf{H}$-noetherian.

Proof. It is clear that $Z \mapsto Z^{+}$is order preserving. By the proposition, we can recover $Z$ from $Z^{+}$, and so the map is injective. Since $\widehat{\mathbf{M}} \times \widehat{\mathbf{E}}$ is the dual of the polynomial representation $\mathbf{M} \oplus \mathbf{E}$ of $\mathbf{G}$, Proposition 5 shows that it is topologically G-noetherian. In particular, the right side of (1) satisfies the descending chain condition. It follows that the left side does as well, and so $\widehat{\mathbf{E}}$ is topologically $\mathbf{H}$ noetherian.

Let $\widehat{\mathbf{U}}^{\prime}$ be the subset of $\widehat{\mathbf{U}}$ consisting of matrices where all diagonal entries are 1, and let $\widehat{\mathbf{W}}=\widehat{\mathbf{U}} \times \widehat{\mathbf{U}}^{\prime}$. Let $\varphi: \widehat{\mathbf{W}} \rightarrow \widehat{\mathbf{M}}$ be the function defined by $\phi(u, v)={ }^{t} u I v$. Note that since ${ }^{t} u \in \widehat{\mathbf{L}}$ and $v \in \widehat{\mathbf{U}}$, this matrix product is defined. Let $\varphi_{n}: \widehat{\mathbf{W}}_{n} \rightarrow$ $\widehat{\mathbf{M}}_{n}$ be defined by the same formula. We note that the diagram

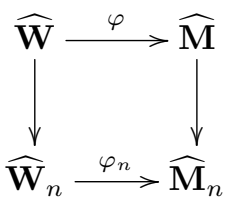

commutes. That is, if $i, j \leq n$ then the $(i, j)$ entry of $\varphi(u, v)$ only depends on the top left $n \times n$ blocks of $u$ and $v$.

Lemma 8. Let $X$ be an affine variety over $\mathbf{k}$, and let $h: \widehat{\mathbf{W}}_{n} \times X \rightarrow \mathbf{k}$ be a regular function. Then there is a monomial $m$ in the diagonal entries of $\widehat{\mathbf{U}}_{n}$ and a regular function $H: \widehat{\mathbf{M}}_{n} \times X \rightarrow \mathbf{k}$ such that $H\left(\varphi_{n}(w), x\right)=m(u) h(w, x)$ holds for all $w=(u, v) \in \widehat{\mathbf{W}}_{n}$ and $x \in X$.

Proof. Consider the polynomial ring

$$
R=\operatorname{Sym}\left(\mathbf{W}_{n}\right) \otimes \mathcal{O}_{X}=\mathbf{k}\left[u_{i j}, v_{k l}\right]_{1 \leq i \leq j \leq n, 1 \leq k<l \leq n} \otimes \mathcal{O}_{X} .
$$

The morphism $\phi_{n}$ induces an injective ring homomorphism $\operatorname{Sym}\left(\mathbf{M}_{n}\right) \otimes \mathcal{O}_{X} \rightarrow R$, so we may view the former as a subring of the latter. Note that this subring is generated (as an $\mathcal{O}_{X}$-algebra) by terms of the form $\sum_{k=1}^{i} u_{k i} v_{k j}$ for $i<j$ and terms of the form $u_{j i}+\sum_{k=1}^{j-1} u_{k i} v_{k j}$ for $i \geq j$. We denote these terms by $a_{i j}$. 
Suppose that we can express all $u_{i j}$ and $v_{k l}$ as a quotient of a polynomial in the $a_{i j}$ by a monomial in the $u_{i i}$. The expression $h(w, x)$ is polynomial in terms of the $u_{i j}$ and $v_{k l}$ (and $x$ ), and we can substitute aforementioned quotients and collect denominators to find an expression of $h(w, x)$ as the quotient of a polynomial in the $a_{i j}$ (and $x$ ) by some monomial in the $u_{i i}$. We let $H$ be the aforementioned polynomial in the $a_{i j}$ and $x$ (a regular function from $\widehat{\mathbf{M}}_{n} \times X \rightarrow \mathbf{k}$ ), and we let $m$ be the corresponding monomial in the $u_{i i}$.

It thus suffices to show that all $u_{i j}$ and $v_{k l}$ can be expressed as a quotient of a polynomial in the $a_{i j}$ by a monomial in the $u_{i i}$. We do so by induction.

We have $u_{1 i}=a_{i 1}$ for any $i \geq 1$ and $v_{1 j}=a_{1 j} / u_{11}$ for any $j>1$. Both of these are expressions of the desired form. Now suppose that $u_{k i}$ and $v_{k j}$ can be expressed in the desired form for all $k \leq i<j$ with $k<K$, for some $K$. For $i \geq K$ we see that $a_{i K}$ is equal to $u_{K i}$ plus terms of the form $u_{k i} v_{k K}$ with $k<K$; since we have an expression of the desired form for each of these other terms, we find one for $u_{K i}$. For $j>K$, we find that $a_{K j}$ is equal to $u_{K K} v_{K j}$ plus terms of the form $u_{k K} v_{k j}$ with $k<K$; once again, this yields an expression of the desired form for $v_{K j}$ by substituting and collecting denominators (which only involve monomials in the $u_{i i}$ ). This concludes the proof of the lemma.

Let $f$ be a regular function on $\widehat{\mathbf{E}}$ of level $n$ that vanishes on $Z$. We define a function $h_{f}$ on $\widehat{\mathbf{W}} \times \widehat{\mathbf{E}}$ by

$$
h_{f}(w, x)=f(w \cdot x) .
$$

Note that the above formula makes use of the action map $\widehat{\mathbf{U}} \times \widehat{\mathbf{U}} \times \widehat{\mathbf{E}} \rightarrow \widehat{\mathbf{E}}$, which exists since $\widehat{\mathbf{E}}$ is a polynomial representation of $\mathbf{G}$.

We claim that $h_{f}$ is a regular function of level $n$. Consider the diagram

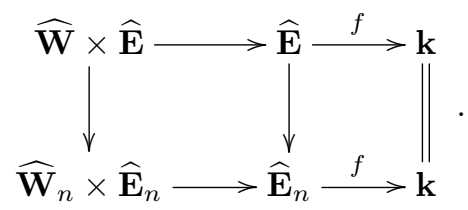

The left horizontal maps are the action maps. Both squares commute, so the whole diagram does, and thus $h_{f}$ factors through the left map and so has level $n$.

It follows from the Lemma 8 that there is a monomial $m_{f}$ in the diagonal entries of $\widehat{\mathbf{U}}_{n}$ such that $m_{f} h_{f}$ induces a regular function on $\widehat{\mathbf{M}} \times \widehat{\mathbf{E}}$ of level $n$; call this function $H_{f}$.

Lemma 9. The function $H_{f}$ vanishes on $Z^{+}$.

Proof. Let $\mathbf{G}_{m}=\mathbf{G L}_{m} \times \mathbf{G L}_{m}$, and let $\mathbf{H}_{m} \subset \mathbf{G}_{m}$ be the set of matrices of the form $\left(g,{ }^{t} g^{-1}\right)$. It suffices to show that $H_{f}$ vanishes on a dense subset of the $\mathbf{G}_{m}$-orbit of $\{I\} \times Z$ for all $m \gg 0$. Now, the set $\mathbf{W}_{m} \mathbf{H}_{m} \subset \mathbf{G}_{m}$ is dense, since a generic element of $\mathbf{G} \mathbf{L}_{m}$ can be written as a product of an upper triangular and lower triangular matrix. Suppose that $g=w h$, with $m \geq n$. Writing $w=(u, v)$, we have $g=\left(u h, v^{t} h^{-1}\right)$. Note that

$$
g \cdot I={ }^{t}(u h)^{-1} I\left(v^{t} h^{-1}\right)^{-1}={ }^{t} u^{-1} I v^{-1}=\varphi\left(w^{-1}\right) .
$$


Let $z \in Z$. We then have

$$
H_{f}(g(I, z))=H_{f}\left(\varphi\left(w^{-1}\right), g z\right)=m_{f}\left(u^{-1}\right) h_{f}\left(w^{-1}, g z\right) .
$$

Now, by definition, we have

$$
h_{f}\left(w^{-1}, g z\right)=f\left(w^{-1} w h z\right)=f(h z) .
$$

Since $Z$ is $\mathbf{H}$-stable, we have $h z \in Z$. Since $f$ vanishes on $Z$, we thus see that this expression vanishes.

Lemma 10. Suppose $z \in \widehat{\mathbf{E}}$ does not belong to $Z$. Then there exists $f$ vanishing on $Z$ such that $H_{f}(I, z) \neq 0$.

Proof. Let $n$ be such that the projection $\bar{z}$ of $z$ to $\widehat{\mathbf{E}}_{n}$ does not belong to $Z_{n}$. We can thus find a regular function $f$ on $\widehat{\mathbf{E}}_{n}$ that vanishes on $Z_{n}$ but does not vanish at $\bar{z}$. We have

$$
H_{f}(I, z)=H_{f}(\varphi(I, I), z)=m_{f}(I) h_{f}((I, I), z)
$$

Since $m_{f}(u)$ is monomial in the diagonal entries of $u$, it follows that $m_{f}(I) \neq 0$. Furthermore, we have $h_{f}((I, I), z)=f(z) \neq 0$. We thus see that $H_{f}(I, z) \neq 0$.

Proof of Proposition 6. It is clear that $\{I\} \times Z$ is contained in the intersection of $\{I\} \times \widehat{\mathbf{E}}$ and $Z^{+}$. Suppose that $z \in \widehat{\mathbf{E}}$ does not belong to $Z$. Let $f$ be as in Lemma 10 . By Lemma 9 , we see that $H_{f}$ belongs to the ideal of $Z^{+}$. Since $H_{f}(I, z) \neq 0$, it follows that $(I, z) \notin Z^{+}$, which proves the result.

\section{Some counterexamples}

Draisma's theorem states that if $\mathbf{E}$ is a polynomial representation of GL then $\widehat{\mathbf{E}}$ is topologically GL-noetherian. One can identify $\mathbf{E}_{n}$ with the spectrum of $\operatorname{Sym}\left(\widehat{\mathbf{E}}_{n}\right)$, and in this way regard $\mathbf{E}=\lim _{\longrightarrow} \mathbf{E}_{n}$ as an ind-scheme. (The elements of $\operatorname{Sym}(\widehat{\mathbf{E}})$ define functions on $\mathbf{E}$, and their zero loci define the closed sets.) It is therefore sensible to ask if $\mathbf{E}$ is topologically $\mathbf{G L}$-noetherian. In [ESS] it is proved that this is the case: in fact, the GL-stable closed sets of $\mathbf{E}$ and $\widehat{\mathbf{E}}$ are shown to be in bijection, and so noetherianity of $\mathbf{E}$ follows from Draisma's theorem.

We now explain that ind-version of Theorem 2 fails. In fact, noetherianity fails due to an obvious obstruction in each case.

First consider the GL case. If $m$ is an element of $\mathbf{M}$ then one can make sense of the determinant $\chi(m)=\operatorname{det}(1-t m)$, a polynomial in $t$. The action of $\mathbf{G L}$ on $\mathbf{M}$ by conjugation defines an algebraic representation, and leaves $\chi$ invariant. Let $c_{i}(m)$ be the coefficient of $t^{i}$ in $\chi(m)$. Then $c_{i}: \mathbf{M} \rightarrow \mathbf{A}^{1}$ is a $\mathbf{G L}$-invariant function. Taken together, the $c_{i}$ define a GL-invariant function $c: \mathbf{M} \rightarrow \mathbf{A}^{\infty}$, where the target is the ind-scheme $\lim \mathbf{A}^{n}$. It is easy to see that $c$ is surjective, from which it follows that $\mathbf{M}$ is not topologically $\mathbf{H}$-noetherian. (If $Z_{\bullet}$ is an infinite strictly descending chain of closed subsets of $\mathbf{A}^{\infty}$ then $c^{-1}\left(Z_{\bullet}\right)$ is an infinite strictly descending chain of $\mathbf{H}$-stable closed subsets of $\mathbf{M}$.) 
The other cases are similar. In the symplectic case, one considers the characteristic polynomial of anti-symmetric matrices, while in the orthogonal case one uses symmetric matrices. We remark that $\widehat{\mathbf{V}}$ (and more generally $\widehat{\mathbf{V}}^{d}$ for any $d \geq 1$ ) is known to be topologically $\mathfrak{S}$-noetherian $[\mathrm{Co}],[\mathrm{Co} 2],[\mathrm{AH}],[\mathrm{HS}]$, where $\mathfrak{S}$ is the infinite symmetric group, and for similar reasons $\mathbf{V}$ is not topologically $\mathfrak{S}$ noetherian (one makes an invariant polynomial by using the coordinates as roots, so that the $c_{i}$ 's are elementary symmetric functions).

It is interesting to observe that these counterexamples do not apply in the pro-setting since the invariants they use no longer make sense: one cannot take the characteristic polynomial of an element of $\overrightarrow{\mathbf{M}}$, as this would involve infinite sums. Similarly, the proof of noetherianity in the pro- setting does not apply in the ind-setting since the element $I \in \widehat{\mathbf{M}}$ does not belong to $\mathbf{M}$.

\section{References}

[AH] M. Aschenbrenner, C. Hillar, Finite generation of symmetric ideals, Trans. Amer. Math. Soc. 359 (2007), 5171-5192.

[AH2] T. Ananyan, M. Hochster, Small subalgebras of polynomial rings and Stillman's conjecture, J. Am. Math. Soc. 33 (2020), 291-309.

[Co] D. E. Cohen, On the laws of a metabelian variety, J. Algebra, 5 (1967), 267-273.

[Co2] D. E. Cohen, Closure relations, Buchberger's algorithm, and polynomials in infinitely many variables, in: Computation Theory and Logic, Lecture Notes in Comput. Sci., Vol. 270, Springer, Berlin, 1987, pp. 78-87.

[CEF] T. Church, J. S. Ellenberg, B. Farb, FI-modules and stability for representations of symmetric groups, Duke Math. J. 164 (2015), no. 9, 1833-1910.

[Dr] J. Draisma, Topological Noetherianity of polynomial functors, J. Am. Math. Soc. 32 (2019), 691-707.

[DE] J. Draisma, R. H. Eggermont, Plücker varieties and higher secants of Sato's Grassmannian, J. Reine Angew. Math. 737 (2018), 189-215.

[DES] H. Derksen, R. H. Eggermont, A. Snowden, Topological noetherianity for cubic polynomials, Algebra \& Number Theory 11 (2017), 2197-2212.

[DK] J. Draisma, J. Kuttler, Bounded-rank tensors are defined in bounded degree, Duke Math. J. 163 (2014), no. 1, 35-63.

[DPS] E. Dan-Cohen, I. Penkov, V. Serganova, A Koszul category of representations of finitary Lie algebras, Adv. Math. 289 (2016), 250-278.

[Eg] R. H. Eggermont, Finiteness properties of congruence classes of infinite matrices, Linear Algebra Appl. 484 (2015), 290-303.

[ESS] D. Erman, S. V. Sam, A. Snowden, Generalizations of Stillman's conjecture via twisted commutative algebras, Int. Math. Res. Not. IMRN, to appear.

[HS] C. Hillar, S. Sullivant, Finite Gröbner bases in infinite dimensional polynomial rings and applications, Adv. in Math. 221 (2012), 1-25.

[NSS] R. Nagpal, S. V. Sam, A. Snowden, Noetherianity of some degree two twisted commutative algebras, Selecta Math. (N.S.), 22 (2016), no. 2, 913-937.

[PSa] A. Putman, S. V. Sam, Representation stability and finite linear groups, Duke Math. J. 166 (2017), 2521-2598. 
[PSe] I. Penkov, V. Serganova, Categories of integrable sl $(\infty)-, o(\infty)-$, sp $(\infty)$-modules, in: Representation Theory and Mathematical Physics, Contemp. Math. 557, AMS, 2011, pp. 335-357.

[PSt] I. Penkov, K. Styrkas, Tensor representations of classical locally finite Lie algebras, in: Developments and Trends in Infinite-dimensional Lie Theory, Progr. Math., Vol. 288, Birkhäuser Boston, Boston, MA, 2011, pp. 127-150.

[Sn] A. Snowden, Syzygies of Segre embeddings and $\Delta$-modules, Duke Math. J. 162 (2013), no. 2, 225-277.

[SS] S. V. Sam, A. Snowden, Stability patterns in representation theory, Forum Math. Sigma 3 (2015), e11, 108 pp.

[SS2] S. V. Sam, A. Snowden, Gröbner methods for representations of combinatorial categories, J. Amer. Math. Soc. 30 (2017), 159-203.

Open Access This article is licensed under a Creative Commons Attribution 4.0 International License, which permits use, sharing, adaptation, distribution and reproduction in any medium or format, as long as you give appropriate credit to the original author(s) and the source, provide a link to the Creative Commons licence, and indicate if changes were made. The images or other third party material in this article are included in the article's Creative Commons licence, unless indicated otherwise in a credit line to the material. If material is not included in the article's Creative Commons licence and your intended use is not permitted by statutory regulation or exceeds the permitted use, you will need to obtain permission directly from the copyright holder. To view a copy of this licence, visit http://creativecommons.org/ licenses/by/4.0/.

Publisher's Note Springer Nature remains neutral with regard to jurisdictional claims in published maps and institutional affiliations. 